\title{
A critical examination of the spectral contrast account of compensation for coarticulation
}

\author{
Navin Viswanathan, Carol A. Fowler, and James S. Magnuson \\ University of Connecticut, Storrs, Connecticut \\ and Haskins Laboratories, New Haven, Connecticut
}

\begin{abstract}
Vocal tract gestures for adjacent phones overlap temporally, rendering the acoustic speech signal highly context dependent. For example, following a segment with an anterior place of articulation, a posterior segment's place of articulation is pulled frontward, and listeners' category boundaries shift appropriately. Some theories assume that listeners perceptually attune or compensate for coarticulatory context. An alternative is that shifts result from spectral contrast. Indeed, shifts occur when speech precursors are replaced by pure tones, frequency matched to the formant offset at the assumed locus of contrast (Lotto \& Kluender, 1998). However, tone analogues differ from natural formants in several ways, raising the possibility that conditions for contrast may not exist in natural speech. When we matched tones to natural formant intensities and trajectories, boundary shifts diminished. When we presented only the critical spectral region of natural speech tokens, no compensation was observed. These results suggest that conditions for spectral contrast do not exist in typical speech.
\end{abstract}

When individuals speak, sequences of vocal tract gestures are coarticulated; the gestures for one phone temporally overlap those for adjacent phones. Coarticulation makes the acoustic manifestations of phones highly context dependent. For example, if a speaker produces the sequence [lg], as in [alga], the point of constriction during $[\mathrm{g}]$ is pulled forward from the velar place of [g] owing to temporal overlap (coproduction) of the anterior constriction for [1]. The point where the tongue hits the palate during the articulation of [g] approximates a weighted vector sum of the constriction locations of the coarticulated segments (Fowler \& Smith, 1986). Thus, during the interval in which [g] has primary but not exclusive control of the vocal tract, the speech signal has an acoustic structure shifted away from canonical [g] (and toward [d]), reflecting the more anterior constriction location. The converse applies when a posterior-anterior sequence is produced, as in [arda].

Mann (1980) found that listeners behave as if they compensate for coarticulation: Category boundaries between phones shift systematically depending on coarticulatory context. For example, the anterior-posterior boundary between [d] and [g] shifts forward after a segment with a posterior place of articulation, and vice versa, suggesting listeners' sensitivity to coarticulation. Two sorts of explanation have been proposed for this phenomenon.

The first posits that perceivers attune to the event of coarticulation (via its acoustic consequences) or to its acoustic correlates themselves. For example, the direct realist theory of speech perception claims that compensation for coarticulation occurs because listeners directly perceive vocal tract gestures and are sensitive to acoustic effects of coarticulation (see Fowler, 2006, for an overview). Thus, for the [alga] example, the direct realist account is that listeners are able to distinguish acoustic information for anterior [1] from that for [g], leading to appropriate context-dependent responses. Mann and Repp (1981) suggested other explanations that also appeal to sensitivity to coarticulation, including a perceptual-learning account on which compensation reflects perceptual experience with acoustic contingencies resulting from coarticulation.

In a second sort of explanation, one does not assume that listeners are directly sensitive to coarticulation. Mann (1980) speculated that sensory contrast based on the relative spectral distribution of energy between the precursor segment and the target might account for compensation effects. Specifically, F3 is relatively high for [1] and [d] but relatively low for [r] and [g]. Hearing the high $F 3$ during [1] might produce a contrastive effect, causing the $F 3$ of the following segment to be heard as lower (and hence more [g]-like). Conversely, hearing a low $F 3$ during the production of [r] might cause a following stop's $F 3$ to be perceived as higher (and hence more [d]-like).

In support of this account, Lotto and Kluender (1998, Experiment 3) found a similar [da]-[ga] boundary shift when natural speech precursors were replaced by pure, steady tones at the $F 3$ offsets of [l] and [r]. These results have been extended to a variety of contexts, including vowel-consonant (VC) coarticulation (Holt, Lotto, \& Kluender, 2000), anticipatory coarticulation (Wade \& Holt, 2005), and effects of speech precursors on percep-

N.Viswanathan, navin.viswanathan@uconn.edu 
tion of nonspeech targets (Stephens \& Holt, 2003). These findings raise questions regarding the interpretation that boundary shifts caused by speech precursors reflect true perceptual compensation for coarticulation. Instead, they seem to suggest that compensation for coarticulation is just another example of the general phenomenon of spectral contrast.

This conclusion is viable only if the acoustic conditions for spectral contrast are present in natural speech. However, this assumption is untested: Indeed, nonspeech precursors have typically differed from the speech formants they model, along three major dimensions. First, tone analogues are typically matched to the intensity of the speech syllable rather than to the formant they represent. Second, steady tones at formant-offset frequencies are typically used instead of tones reflecting formant transitions. Third, the harmonic structure of the critical formant is not captured by the sine-wave tone analogues (cf. Holt, 1999).

There is preliminary evidence that matching tones along these dimensions might affect the potency of contrast effects. Lotto and Kluender (1998, Experiment 2) found that sine-wave tones tracking the transitions of the speech syllables and intensity matched to $F 3$ region $^{1}$ (matched along two of three dimensions of the differences listed above) produced numerically smaller effects than did the original speech syllables. In contrast, effects with steady tones matched to entire syllable intensity generally led to effect magnitudes comparable to those observed with natural speech. However, Lotto and Kluender did not report statistical tests of the different effect magnitudes. Similarly, Mitterer (2006, Experiment 2B) investigated whether sinewave analogues of fricatives were sufficient to produce contrastive effects on judgments of a following vowel. He used steady-tone analogues of fricatives, but with intensities matched to $F 3$, and failed to find contrast effects. We will directly test whether compensation for coarticulation with natural speech can be attributed to spectral contrast, by examining whether the conditions for spectral contrast are provided by natural speech materials.

From a spectral contrast perspective (e.g., Lotto \& Kluender, 1998), compensation for coarticulation in the disyllables used by Mann (1980) is due to the spectrally contrastive nature of their third formant transitions. We tested this explanation directly by providing only the $F 3$ region of natural speech syllables as precursors in Experiment 1. If the conditions for spectral contrast for the Mann items are provided by $F 3$ in natural speech, the critical region by itself should be as effective as the whole speech syllable in inducing boundary shifts.

\section{EXPERIMENT 1}

Two groups identified syllables from a [da]-[ga] continuum. For one group, the stops were preceded by natural tokens of [al] or [ar], to replicate compensation studies that used natural speech precursors. Another group heard precursors only including the critical $F 3$ region of these syllables, providing a direct test of whether a natural $F 3$ provides conditions for spectral contrast.

\section{Method}

Participants. Forty-four University of Connecticut undergraduates (22 in each group) participated for course credit. All reported normal hearing.

Materials. We created an 11-step continuum of resynthesized $\mathrm{CV}$ syllables, varying in $F 3$-onset frequency and varying perceptually from [da] to [ga], via the source-filter method using Praat (Boersma, 2001). F3-onset frequencies varied in 100-Hz steps from $1800 \mathrm{~Hz}$ ([ga]) to $2800 \mathrm{~Hz}$ ([da]), changing linearly to a steady-state value of $2500 \mathrm{~Hz}$ over an 80 -msec transition. The first, second, and fourth formants were the same for all members of the continuum. Over the 80 -msec transition, $F 1$ shifted from 500 to $800 \mathrm{~Hz}, F 2$ shifted from 1600 to $1200 \mathrm{~Hz}$, and $F 4$ was held steady at $3500 \mathrm{~Hz}$. The overall duration of each CV syllable was $215 \mathrm{msec}$.

In one condition, stops were preceded by natural tokens of [al] and [ar]. The precursors were matched in duration $(375 \mathrm{msec})$ and intensity. The critical $F 3$ offsets were approximately $2600 \mathrm{~Hz}$ for [al] and $1820 \mathrm{~Hz}$ for [ar]. $F 2$ and $F 4$ offsets were approximately 930 and $3530 \mathrm{~Hz}$, respectively, for [al] and 1400 and $3050 \mathrm{~Hz}$, respectively, for [ar]. These acoustic characteristics are similar to those of materials used in previous studies (e.g., Lotto \& Kluender, 1998).

In the second condition, the precursors were filtered to isolate the critical $F 3$ region of the natural syllables. We used a Hanning bandpass filter passing frequencies between 1600 and $3000 \mathrm{~Hz}$ with a smoothing of $100 \mathrm{~Hz}$ to isolate the $F 3$ region. The frequency window was selected by examining the acoustic profile of the natural syllables and ensuring that the $F 3$ information in each liquid was preserved even though we used the same window for both syllables.

VC precursors and CV targets were combined, separated by a silent gap of $50 \mathrm{msec}$, to form 22 unique disyllables (2 precursors $\times$ 11 targets) per group. The stimuli were presented at an 11-kHz sampling rate with 16-bit resolution. Spectrograms of the precursors in each condition with a sample target syllable are shown in Figures 1A and $1 \mathrm{~B}$.

Procedure. We used a two-alternative forced choice task: Participants pressed keys labeled "d" or "g" to indicate their identification of the target. There were two blocks of trials. The first block consisted of practice trials presenting only the [da] and [ga] endpoints with feedback. There were 12 trials with each endpoint, presented in random order. This block familiarized participants with the task and target syllables and provided a basis for ensuring that they could identify the endpoints accurately.

In the second block, each of the 22 disyllables was presented eight times, resulting in 176 trials. No feedback was provided in this block.

\section{Results}

We excluded data from 2 participants in each condition with accuracy less than $80 \%^{2}$ in the endpoint identification block, leaving 20 participants in each condition. The data from the second block were submitted to a 2 (groups) $\times 2$ (precursor) $\times 11$ (step) mixed ANOVA. The main effect of precursor was significant $[F(1,38)=$ $\left.20.20, p<.001, \eta_{\mathrm{p}}^{2}=.35\right]$, with more "g" responses following [al] precursors $(51.3 \%)$ than following [ar] precursors $(47.3 \%$; see Figures 1A and 1B). The main effect of group was not significant $\left(F<1, \eta_{\mathrm{p}}^{2}=.02\right)$. Most critically, there was a strong interaction between precursor and group $\left[F(1,38)=16.38, p<.001, \eta_{\mathrm{p}}^{2}=.30\right]$, suggesting a difference in compensation across precursor conditions. Planned contrasts confirmed that the natural syllables produced the expected compensation effect $(7.6 \%$ more "g" responses following [al] than following [ar]) $[F(1,19)=$ $\left.32.70, p<.001, \eta_{\mathrm{p}}^{2}=.63\right]$, replicating earlier studies (e.g., Mann, 1980); however, the ostensible critical region alone did not produce a compensation-like effect (only $0.4 \%$ 


\section{Precursors With Example \\ Target Syllable}

A [al] or [al] Analogues

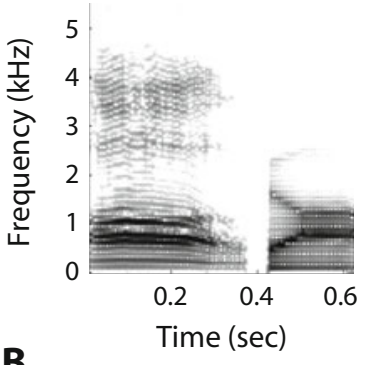

B

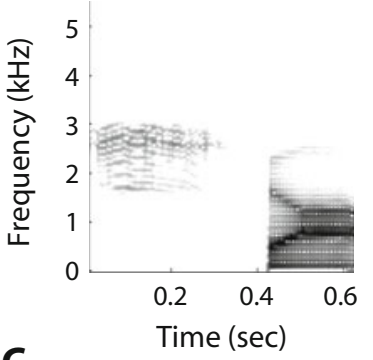

C

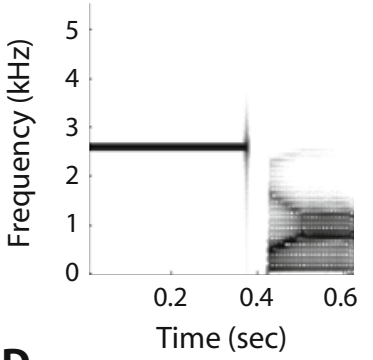

D

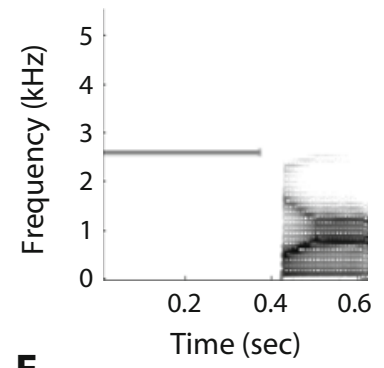

E

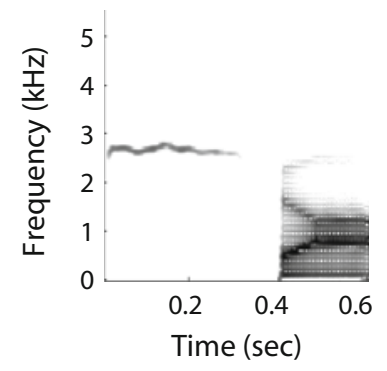

[ar] or [ar] Analogues
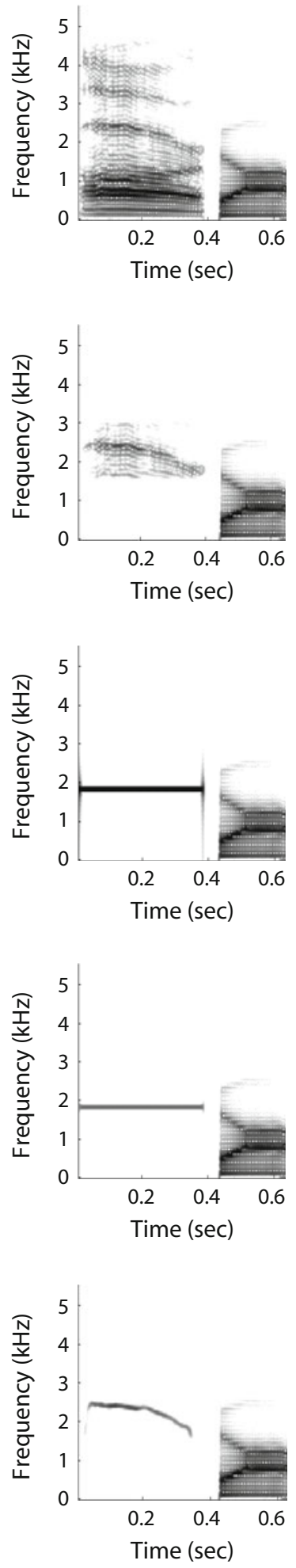

Effect on Following Stop Judgment
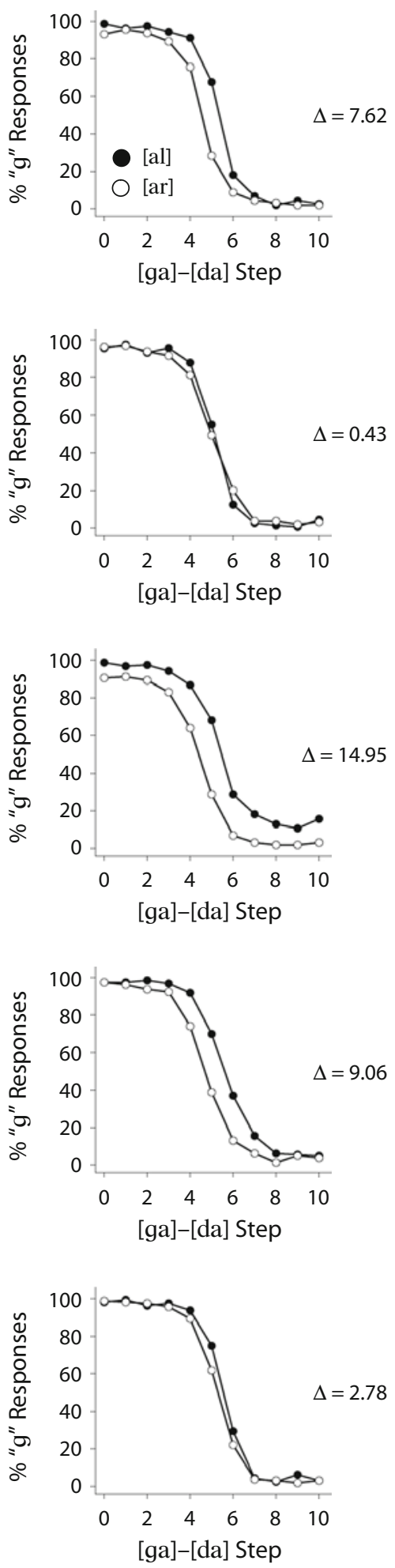

Figure 1. Spectrograms of representative precursors before sample target syllable in each condition. Precursors and results of Experiment 1 are depicted in panels $A$ and $B$ and of Experiment 2 in panels $C-E$. Filled circles indicate responses to $[\mathrm{al}]$ or [al] analogues, and open circles indicate responses to [ar] or [ar] analogues. $\Delta$ indicates the average percentage shift in categorization caused by the precursors, averaged over participants and step. 
more "g" responses following the $F 3$ region of [al] than following that of [ar] $)\left(F<1, \eta_{\mathrm{p}}^{2}=.07\right)$. Although the absence of a reliable shift with filtered precursors must be interpreted with caution, these items did contain the $F 3$ region of the very syllable precursors that produced a strong boundary shift effect. Moreover, any reduction of the effect (let alone the absence observed here) sufficient to yield a reliable interaction is problematic for a contrast account in which compensation for coarticulation is directly attributed to the contrastive effects of precisely the critical region that these precursors isolate.

We designed Experiment 2 to investigate why, despite robust boundary-shift effects following single-formant tone analogues matched to $F 3$ (e.g., Lotto \& Kluender, 1998), the filtered $F 3$ region of speech by itself does not produce category shifts. Perhaps the higher intensity and energy concentration at the offset frequency in typical tone analogues, relative to the critical $F 3$ region of natural speech, provide the conditions needed for spectral contrast. If so, contrast effects should diminish when tones are matched to additional natural formant characteristics. We tested this hypothesis in Experiment 2.

\section{EXPERIMENT 2}

Three groups participated in this experiment. One group heard typical steady-tone analogue precursors matched in frequency to $F 3$ offsets and matched to the intensity of whole syllables (as in Lotto \& Kluender, 1998, Experiment 3 ). The precursors for the second group were the same, except that intensity was matched to the critical formant rather than to the whole syllable. The precursors for the third group were matched in both intensity and trajectory to the critical third formant. If steady, high-intensity tones provide the strongest conditions for spectral contrast, we should find that effects weaken as tones are progressively matched to additional $F 3$ characteristics.

\section{Method}

Participants. Sixty-eight University of Connecticut undergraduates (24 in Group 1, 21 in Group 2, and 23 in Group 3), who reported normal hearing, participated for course credit. None had participated in Experiment 1.

Materials. The [da]-[ga] continuum from Experiment 1 was used. Two steady-state sine-wave tones were used as precursors for the first group: a high $F 3$ tone at $2600 \mathrm{~Hz}$ ( $F 3$ offset of [1]) and a low $F 3$ tone at $1820 \mathrm{~Hz}$ ( $F 3$ offset of [ar]). Following Lotto and Kluender (1998, Experiment 3), the intensities and durations of the precursor tones were matched to the overall intensities and durations of the precursor syllables used in Experiment 1.

The second group heard steady tones at the same frequencies as did Group 1, with a crucial difference: Tone intensities were matched to the intensity of the formant that they were designed to represent (as in Mitterer, 2006, Experiment 2B). The intensity of the higher frequency [al] tone was set to $48 \mathrm{~dB}$ and the lower frequency tone to $52 \mathrm{~dB}$, obtained by measuring the intensities of the filtered [al] and [ar] critical regions used in Experiment 1.

The third group heard tonal analogues with intensities and trajectories matched to those of the critical formant from the syllables that they were designed to represent. These tones were generated by tracking the center frequency of the formants in the natural speech tokens used in Experiment 1.

Procedure. The procedure of Experiment 1 was used.

\section{Results}

We excluded 8 participants with accuracy less than $80 \%$ in endpoint identification, leaving 20 participants in each condition. Data from the second block (shown in Figures $1 \mathrm{C}, 1 \mathrm{D}$, and $1 \mathrm{E}$ ) were submitted to a 3 (group) $\times 2$ (precursor) $\times 11$ (step) mixed ANOVA. The main effect of precursor was significant $\left[F(1,57)=50.18, p<.001, \eta_{\mathrm{p}}^{2}=\right.$ .47], since there were more "g" responses following [al] analogues $(56.3 \%)$ than following [ar] analogues $(47.4 \%)$. The effect of group was not significant $[F(2,57)=2.113$, $\left.p=.13, \eta_{\mathrm{p}}^{2}=.06\right]$. However, the critical group $\times$ precursor interaction was significant $\left[F(1,57)=7.83, p=.001, \eta_{\mathrm{p}}^{2}=\right.$ .22 ], with the effect of precursor diminishing (see Figure 2) as precursors were incrementally matched to conditions in speech. Pairwise comparisons indicated that tones matched to $F 3$ in both their frequency trajectory and intensity produced smaller effects than did syllable-intensity steady tones at critical formant offsets ( $p=.045$ ). Other comparisons were not significant. A comparative analysis across both experiments is reported at the end of this section.

Although these findings agree in pattern with those of past studies using liquid-stop contexts (Lotto \& Kluender, 1998, Experiments 2 and 3), they do not appear to generalize to a consonant-vowel-consonant context. For instance, Holt et al. (2000) used tone analogues matching $F 2$ formant trajectory (the origin of contrast in this context) that appear to produce larger effects than do steady tones at $F 2$ offset. To explain this difference, Holt et al. (2000) speculated that perhaps steady tones placed at formant offsets (similar to those in our study and to those of Lotto \& Kluender's [1998] Experiment 3) would produce weaker effects on vowel categorization than would those placed at the center frequency of the critical formant.

We agree that the specific choice of frequency for steady-tone analogues is likely to be crucial in affecting the magnitude and occurrence of contrast effects across different coarticulatory contexts. One approach, as Holt et al. (2000) suggested, is to construct an empirically based psychoacoustic model to guide this decision. However, a more direct approach is to test the critical region claimed to be causal, as we did in Experiment 1 (by filtering to isolate the critical region). Following this approach, one can be sure neither to underestimate nor to overestimate the conditions present in natural speech for spectral contrast.

\section{OMNIBUS ANALYSIS}

We conducted an omnibus analysis to compare results across conditions in the two experiments. We calculated the amount of compensation by subtracting the mean percentage of " $\mathrm{g}$ " responses to [ar] (or, in nonspeech conditions, its analogue) from the mean percentage of " $g$ " responses to [al] (or its analogue), at each step of the continuum, for each participant. The results for each condition are shown in Figure 2. The figure suggests that typically used nonspeech tones yield the numerically largest contrast effects, and, as these analogues are made more like speech, their effects diminish.

We submitted the difference scores to a 5 (condition) $\times$ 11 (step) mixed ANOVA. The effect of condition was sig- 


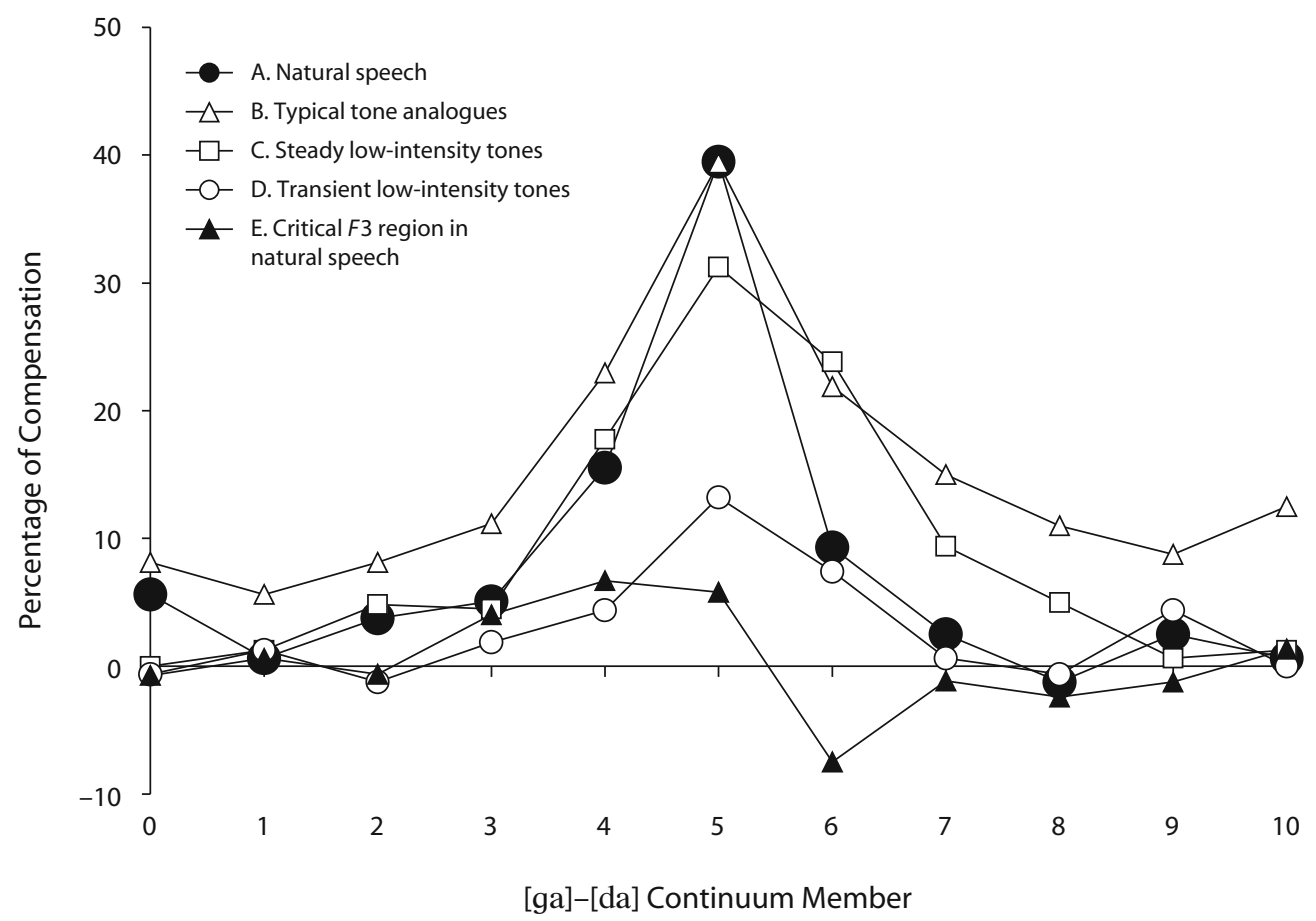

Figure 2. Across-experiment comparison of the amount of compensation as a function of relatedness of tone analogues to speech. As tones are incrementally matched to conditions in speech, contrast effects diminish.

nificant $\left[F(4,95)=9.19, p<.001, \eta_{\mathrm{p}}^{2}=.28\right]$, indicating that the amount of compensation varied across types of precursors. The effect of step $[F(10,950)=24.38, p<$ $\left..001, \eta_{\mathrm{p}}^{2}=.20\right]$ was also significant, because compensation varied across the continuum. We conducted planned comparisons on the amount of compensation in the speech condition $(7.6 \%)$ versus that with each analogue pair. There was greater compensation for the speech condition than for intensity-matched transient tones [2.8\% compensation; $\left.F(1,38)=9.92, p<.003, \eta_{\mathrm{p}}^{2}=.21\right]$ or for the critical region alone $[0.4 \%$ compensation; $F(1,38)=16.22$, $\left.p<.001, \eta_{\mathrm{p}}^{2}=.30\right]$. Compensation in the typical steadytone condition $(15 \%)$ was marginally greater than in the speech condition $\left[F(1,38)=4.07, p=.051, \eta_{\mathrm{p}}^{2}=.10\right]$. The difference between formant-intensity steady tones ( $9.1 \%$ compensation) and the speech condition was not reliable $\left(F<1, \eta_{\mathrm{p}}^{2}=.01\right)$.

\section{GENERAL DISCUSSION}

Whereas other accounts of the phenomenon of compensation for coarticulation have appealed to attunement to acoustic information for coarticulation itself (Fowler, 2006) or to learning acoustic contingencies that coarticulation causes (Mann \& Repp, 1981), the spectral contrast account proposes that compensation is a result of a general contrast phenomenon. As Mann (1980) pointed out, $F 3$ differences between [r] and [l] relative to those of [d] and $[\mathrm{g}]$ correlate with the boundary shifts observed in [d]-[g] identification in the context of the liquids. When Lotto and Kluender (1998) first tested this hypothesis, their evidence appeared clear: Pure tones matched in frequency to the $F 3$ offsets of [ar] and [al] had effects on [d]-[g] identification similar to those of natural tokens of [ar] and [al]. Such results seem to imply that contrast between liquid and stop $F 3$ frequencies was the likely cause of boundary shifts observed with natural speech precursors. However, the validity of this account requires that energy in natural liquid formants provides the conditions for spectral contrast. Heretofore, this has not been tested.

In Experiment 1, we replicated compensation for coarticulation with natural speech precursors. To directly test whether the $F 3$ region of the natural precursors provides a sufficient basis for spectral contrast, we filtered the natural precursors such that only the $F 3$ region remained. Listeners presented with these materials did not exhibit compensation for coarticulation. In Experiment 2, we found that, as tone precursors are made progressively more like speech, contrast effects diminish. In fact, the tone analogues most unlike speech (high-intensity steady tones of the sort typically used in contrast studies) produced responses similar in magnitude to those elicited by speech. We found progressively weaker results as tones were matched in intensity and trajectory to the formant they modeled, as compared with steady, syllable-intensity tones.

The most direct implication of the absence of compensation given $F 3$ alone is that compensation effects obtained with speech syllables cannot be attributed to the spectrally contrastive effects of a natural speech precursor's F3. Moreover, compensation-like boundary shifts found in typical contrast experiments using tones may fol- 
low from characteristics of tone precursors that are unlike relevant characteristics of natural speech. This implies that compensation-like shifts following tone precursors that appear to indicate spectral contrast cannot be automatically generalized to effects in natural speech. Thus, the present results pose a substantial challenge for the spectral contrast account of compensation for coarticulation. Our results do not provide direct evidence for accounts that explicitly posit compensation for coarticulation (e.g., direct realism, Fowler, 2006; or perceptual learning, Mann \& Repp, 1981). These coarticulatory accounts do not directly address effects of tonal precursors on speech categorization. On the other hand, the spectral contrast account provides clear, testable predictions concerning boundary shifts that follow such tonal precursors.

Similar responses in tonal and speech conditions in compensation experiments need not imply identical bases of these effects (cf. Fowler, 1990). Indeed, compensation for coarticulation occurs in cases in which no spectrally contrastive segments are present. For example, precursor differences leading to compensation effects may be visually specified (Fowler, Brown, \& Mann, 2000; Mitterer, 2006, Experiment 3; for a debate about visual influences on compensation, see Fowler, 2006; Holt, Stephens, \& Lotto, 2005; Lotto \& Holt, 2006). In addition, compensation occurs when coarticulating gestures are cotemporal (e.g., Silverman, 1986), in the absence of contrastive contexts.

Furthermore, in a recent study, we dissociated the effects of place of articulation and $F 3$ in a liquid precursor context (Viswanathan, Magnuson, \& Fowler, 2008). We utilized a trilled liquid from Tamil (classified by American English listeners as "r") with an alveolar place of articulation (comparable to the English /1/) but a low F3 (comparable to the English / $/ \mathrm{r} /$ ). Our listeners' responses to this Tamil liquid patterned with the English /1/, which shared a place of articulation, rather than with the English $/$ r/, despite its similarly low $F 3$ offset. Thus, when $F 3$ and the place of articulation of the precursor are dissociated, listeners appear to compensate according to the place of articulation - even in a direction opposite to that predicted by sensory contrast.

In summary, compensation for coarticulation occurs both in the direction predicted by spectral contrast and in the opposite direction, and it occurs in the absence of spectrally contrastive relations. Moreover, even when the direction of compensation is in the direction predicted by spectral contrast - as is the case of Experiment 1 - the region assumed to provide conditions for contrast is insufficient to produce compensation. Given these dissociations, the general claim that spectral contrast underlies compensation for coarticulation appears unwarranted.

\section{AUTHOR NOTE}

This research was supported by NSF Grant 0642300 to J.S.M., C.A.F., and N.V.; NIH Grant DC00565 to J.S.M.; and NIH Grant HD01994 to Haskins Laboratories. We thank Mark Pitt, Steve Goldinger, and an anonymous reviewer for valuable comments. Correspondence concerning this article should be addressed to N. Viswanathan, Department of Psychology, University of Connecticut, 406 Babbidge Road, Unit 1020, Storrs, CT 06269-1020 (e-mail: navin.viswanathan@uconn.edu).

\section{REFERENCES}

Boersma, P. (2001). Praat, a system for doing phonetics by computer. Glot International, 5, 341-345.

FowLER, C. A. (1990). Sound-producing sources as objects of perception: Rate normalization and nonspeech perception. Journal of the Acoustical Society of America, 88, 1236-1249.

Fowler, C. A. (2006). Compensation for coarticulation reflects gesture perception, not spectral contrast. Perception \& Psychophysics, 68, 161-177.

Fowler, C. A., Brown, J., \& ManN, V. (2000). Contrast effects do not underlie effects of preceding liquid consonants on stop identification in humans. Journal of Experimental Psychology: Human Perception \& Performance, 26, 877-888.

Fowler, C. A., \& SMITH, M. R. (1986). Speech perception as "vector analysis": An approach to the problems of invariance and segmentation. In J. S. Perkell \& D. H. Klatts (Eds.), Invariance and variability in speech processes (pp. 123-139). Hillsdale, NJ: Erlbaum.

Holt, L. L. (1999). Auditory constraints on speech perception: An examination of spectral contrast. Unpublished doctoral dissertation, University of Wisconsin, Madison.

Holt, L. L., Lotto, A. J., \& Kluender, K. R. (2000). Neighboring spectral content influences vowel identification. Journal of the Acoustical Society of America, 108, 710-722.

Holt, L. L., Stephens, J. D., \& LotTo, A. J. (2005). A critical evaluation of visually moderated phonetic context effects. Perception \& Psychophysics, 67, 1102-1112.

LotTo, A. J., \& Holt, L. L. (2006). Putting phonetic context effects into context: A commentary on Fowler (2006). Perception \& Psychophysics, 68, 178-183.

Lotto, A. J., \& KLuender, K. R. (1998). General contrast effects of speech perception: Effect of preceding liquid on stop consonant identification. Perception \& Psychophysics, 60, 602-619.

MANN, V. A. (1980). Influence of preceding liquid on stop-consonant perception. Perception \& Psychophysics, 28, 407-412.

MANN, V. A., \& REPP, B. H. (1981). Influence of preceding fricative on stop consonant perception. Journal of the Acoustical Society of America, 69, 548-558

MitTerer, H. (2006). On the causes of compensation for coarticulation: Evidence for phonological mediation. Perception \& Psychophysics, 68, 1227-1240.

Silverman, K. (1986). $\mathrm{F}_{0}$ segmental cues depend on intonation: The case of the rise after voiced stops. Phonetica, 43, 76-92.

Stephens, J. D. W., \& Holt, L. L. (2003). Preceding phonetic context affects perception of nonspeech. Journal of the Acoustical Society of America, 114, 3036-3039.

Viswanathan, N., Magnuson, J. S., \& Fowler, C. A. (2008). Compensation for coarticulation: Disentangling auditory and gestural theories of perception of coarticulatory effects in speech. Manuscript submitted for publication.

WADE, T., \& HOLT, L. L. (2005). Effects of later-occurring non-linguistic sounds on speech categorization. Journal of the Acoustical Society of America, 118, 1701-1710.

\section{NOTES}

1. Lotto and Kluender (1998) used tones modeling the formant transitions alone ( $F 3$ glides), whereas our tones reflected both the steady and transient parts of $F 3$.

2. Using a cutoff of $90 \%$ (cf. Lotto \& Kluender, 1998) did not qualitatively change our results.

(Manuscript received April 14, 2008; revision accepted for publication August 28, 2008.) 